\title{
Wing length and host location in tsetse (Glossina spp.): implications for control using stationary baits
}

John Hargrove ${ }^{1^{*}}$ (D), Sinead English², Stephen J. Torr ${ }^{3}$, Jennifer Lord ${ }^{3}$, Lee Rafuse Haines ${ }^{3}$, Cari van Schalkwyk', James Patterson ${ }^{4}$ and Glyn Vale ${ }^{1,5}$

\begin{abstract}
Background: It has been suggested that attempts to eradicate populations of tsetse (Glossina spp.) using stationary targets might fail because smaller, less mobile individuals are unlikely to be killed by the targets. If true, tsetse caught in stationary traps should be larger than those from mobile baits, which require less mobility on the part of the flies.

Results: Sampling tsetse in the Zambezi Valley of Zimbabwe, we found that the number of tsetse caught from stationary traps, as a percent of total numbers from traps plus a mobile vehicle, was $\sim 5 \%$ for male G. morsitans morsitans (mean wing length $5.830 \mathrm{~mm}$; 95\% Cl: 5.800-5.859 mm) and 10\% for females (6.334 mm; 95\% Cl: 6.329$6.338 \mathrm{~mm})$; for G. pallidipes the figures were $~ 50 \%$ for males $(6.830 \mathrm{~mm} ; 95 \% \mathrm{Cl}: 6.821-6.838 \mathrm{~mm})$ and 75\% for females (7.303 mm, 95\% Cl: 7.302-7.305 mm). As expected, flies of the smaller species (and the smaller sex) were less likely to be captured using stationary, rather than mobile sampling devices. For flies of a given sex and species the situation was more complex. Multivariable analysis showed that, for females of both species, wing lengths changed with ovarian age and the month, year and method of capture. For G. pallidipes, there were statistically significant interactions between ovarian age and capture month, year and method. For G. m. morsitans, there was only a significant interaction between ovarian age and capture month. The effect of capture method was, however, small in absolute terms: for G. pallidipes and G. m. morsitans flies caught on the mobile vehicle had wings only 0.24 and $0.48 \%$ shorter, respectively, than flies caught in stationary traps. In summary, wing length in field samples of tsetse varies with ovarian age, capture month and year and, weakly, with capture method. Suggestions that a target-based operation against G. f. fuscipes in Kenya caused a shift towards a smaller, less mobile population of tsetse, unavailable to the targets, failed to account for factors other than capture method.
\end{abstract}

Conclusions: The results are consistent with the successful use of targets to eradicate populations of tsetse in Zimbabwe. Until further, more nuanced, studies are conducted, it is premature to conclude that targets alone could not, similarly, be used to eradicate G. f. fuscipes populations in Kenya.

Keywords: Tsetse Glossina, Eradication using targets, Stationary and mobile baits, Wing length, Age season annual effects

\footnotetext{
* Correspondence: jhargrove@sun.ac.za

'SACEMA, University of Stellenbosch, Stellenbosch, South Africa

Full list of author information is available at the end of the article
}

(c) The Author(s). 2019 Open Access This article is distributed under the terms of the Creative Commons Attribution 4.0 International License (http://creativecommons.org/licenses/by/4.0/), which permits unrestricted use, distribution, and reproduction in any medium, provided you give appropriate credit to the original author(s) and the source, provide a link to the Creative Commons license, and indicate if changes were made. The Creative Commons Public Domain Dedication waiver (http://creativecommons.org/publicdomain/zero/1.0/) applies to the data made available in this article, unless otherwise stated. 


\section{Background}

The host-orientated behaviour of tsetse (Glossina spp.) is important in determining the epidemiology of the potentially fatal diseases caused by the blood parasites (Trypanosoma spp.) which male and female tsetse can transmit when feeding on humans and livestock [1-4]. Vale et al. [5] suggested that the distinctive behaviour patterns of different tsetse species, and of the two sexes, are markedly affected by the mobility of the flies, which is increased when the habitat is extensive and flies are large. The sorts of behaviour affected include: (i) the relative probability of being caught by stationary as against mobile baits; (ii) the reliance on odours rather than visual stimuli in host detection; (iii) the probability of alighting on vertebrate hosts, or artificial baits; and (iv) the species of host selected. Each of these behavioural effects is subject to various, sometimes competing, considerations.

On one hand, if a fly stays still, it can find only mobile hosts that pass by, whereas the more it moves the greater the chance that it will discover more hosts of any sort, but especially more stationary hosts. The greater the number of hosts discovered, the more the flies can afford to be selective in the type of host on which they attempt to feed. Close-range responses by the fly can also be geared to making the appropriate selection, as against alighting and probing at the first opportunity in high risk situations, for example if there are bembicid wasps or asilid (robber) flies in attendance, or if the potential host itself is likely to kill the fly, as in the case of humans and baboons.

On the other hand, active searching increases the rate at which energy is utilised by perhaps 100 -fold [6]. It is reasonable to suppose that considerations of energy conservation might be more serious for smaller species of flies, in accord with the findings of Vale et al. [5]. The two host-locating options are not mutually exclusive: Brady's laboratory experiments $[7,8]$ showed that both spontaneous activity and responses to a mobile object increase in intensity with time since last feeding. Tsetse may feed off mobile baits early in the hunger cycle, if the feeding risk is low and little flight activity is required $[9,10]$. Later in the hunger cycle, having failed to feed off a passing host, flies show higher levels of spontaneous activity and have a relatively higher probability of locating hosts.

Vale et al. [5] did not consider whether, within a given sex and species, host-orientated behaviour patterns might also be a function of fly size. Such an effect has, however, been suggested for Glossina fuscipes fuscipes Newstead in Kenya [11]. The core evidence was that, prior to a 19-month control campaign using stationary insecticide-treated targets on Big Chamaunga Island [12], the wing sizes of sampled females were $1 \%$ larger than they were four years later.
It was suggested that: (i) targets selectively killed larger flies within the initial population; (ii) this selection in favour of genetically and phenotypically smaller, less mobile, flies might have left a remnant population that could not be eradicated using targets; (iii) such an effect might explain the claimed rarity of reports of the successful elimination of tsetse populations using targets alone [13, 14]; and (iv) the change in fly size could alter the epidemiology of tsetse-borne diseases by modifying the patterns of feeding.

The above suggestions lead to the testable hypothesis that tsetse of a given sex and species captured at a stationary bait should be larger, on average, than those captured at a mobile bait, since the latter requires a lesser demand on the flies' flight capability. The hypothesis would become more convincing if the size variation due to bait type were great in relation to the variations associated with different taxa and with non-genetic background factors that are not related to control campaigns. To test this hypothesis, we studied the numbers and mean wing lengths of male and female G. m. morsitans Westwood and G. pallidipes Austen, sampled at Rekomitjie Research Station, Zimbabwe, using stationary odour-baited traps and a mobile vehicle-mounted electric target. We first investigated whether, indeed, the relative probability of capture by mobile and stationary baits differed between the sexes and species, according to mean wing length. Next, we assessed whether the capture probability also differed between the larger and smaller individuals within a single taxon. Finally, we explored the background variations in size due to season, year and fly age and interactions between these factors.

\section{Methods}

The study was carried out at Rekomitjie Research Station, Zambezi Valley, Zimbabwe $\left(16^{\circ} 10^{\prime} \mathrm{S}, 29^{\circ} 25^{\prime} \mathrm{E}\right.$, altitude approximately $520 \mathrm{~m}$ ). The station is located in the Mana Pools National Park, which, together with the Sapi, Hurungwe and Chewore Safari Areas, has a total area of nearly $10,000 \mathrm{~km}^{2}$. Since 1958 there has been no agricultural settlement in this area, which was designated a UNESCO World Heritage Site in 1984, and is protected against settlement, agriculture, illegal hunting and logging. There has been no form of tsetse control in the area, nor has the area been subject to other deliberate environmental or sociological change since it was declared a National Park. Analyses of vegetation cover [15] and of game numbers [16] suggest that the Rekomitjie area continues to provide highly suitable habitat for tsetse.

The data used in this study form a subset of the results of an 11-year sampling study carried out between September 1988 and December 1999 [15]. The subset consists of flies captured in the 6-year period from January 1989 to 
December 1994, chosen because these were the only years when flies were captured using both stationary and mobile sampling devices. The particular devices used for the main part of the study were: (i) stationary mechanical 'epsilon' traps [17] baited with artificial host-odour consisting of acetone, 1-octen-3-ol, 3-n-propyl phenol and 4-methyl phenol released at $\sim 200,0.4,0.01$ and $0.8 \mathrm{mg} / \mathrm{h}$, respectively; and (ii) a vehicle-mounted electric target (VET), which consisted of an electrocuting grid, $1 \mathrm{~m}$ tall and $2 \mathrm{~m}$ long [18] mounted on the back of an open pickup truck [19]. For brevity these two systems are referred to below as "trap" and "VET", respectively. For further details of these sampling devices, and of artificial refuges used in the collection of ancillary data, see Additional file 1: Figures S1-S3 and associated text in Additional file 1: Text S1.

Daily maximum and minimum temperatures were recorded from mercury thermometers housed in a Stevenson screen at the Research Station. Daily rainfall was recorded using a gauge placed $4 \mathrm{~m}$ from the Stevenson screen.

Details of the tsetse life-cycle are provided in Additional file 1: Text S2. The total numbers of each sex and species of tsetse caught by each bait were recorded, but wing length was measured in only a random sample of captured flies. Captured flies, after transfer to the laboratory, were placed in individual $(75 \times 25) \mathrm{mm}$ plastic tubes and placed under a black cloth to reduce activity. Females were then subjected to ovarian dissection, using the technique developed for tsetse by Saunders $[20,21]$ and improved by Challier [22, 23]. The procedure is described in detail by Hargrove [24] and in Additional file 1: Figure S4 and associated text in Additional file 1: Text S3. Technicians dissected flies in the order that they were provided. The ovarian dissection technique is used to gauge the number of times a female fly has ovulated, and thus provides a measure of her age. For studies of wing length, as indices of body size, wings of sampled flies were affixed with transparent sticky tape to the dissection record form and a measure of wing length (as explained in further detail below) was taken using a binocular microscope fitted with a graduated reticule in the eyepiece. Sub-samples of 979 female G. pallidipes caught in artificial refuges in October 1992 [25, 26] and 1752 in odour-baited traps in February 1994 [27] were subjected both to ovarian dissection and, thereafter, to nutritional analysis, to ascertain levels of fat and of the residual (i.e. fat-free) dry weights of the thorax and abdomen [26, 27]. Tsetse enter refuges only when temperatures exceed $32{ }^{\circ} \mathrm{C}$, generally in September through November. The construction and use of the artificial refuges are fully described elsewhere [25, 26]. Climate profiles for Rekomitjie Research Station are discussed in Additional file 1: Text S4 and monthly mean maximum and minimum temperatures and rainfall are illustrated in Additional file 1: Figure S5.

C. H. N. Jackson instituted the practice of measuring the length of the middle part of the fourth longitudinal vein (length $C D$, Fig. 1), corresponding to the "cutting blade" of the hatchet cell and this practice has been followed by most tsetse workers in the past [28]. We used, instead, the distance between markers A and B (Fig. 1) because this is a longer distance, so that the relative error, arising as a result of variation about the choice of endpoints due to vein thickness, should be reduced. Measurements, all made on a single wing of each fly, were calibrated using a stage micrometer and all lengths were converted to $\mathrm{mm}$.

It should be noted that Mbewe et al. [11] used, as a measure of fly size, the "centroid size" (CS) defined by Dujardin \& Slice [29] as the square root of the sum of the squared distances to the centroid of each of a number of pre-defined landmarks on a wing. We have data for these landmarks for a subset of 40 wings collected in January 1994 and we compared the CS values obtained for these data with our measure of the wing lengths. We also investigated the correlation between our measure of wing length and the thoracic residual (fat free) dry weight, which has been used previously as a measure of size in tsetse [30]. These correlations are demonstrated using sub-samples of female G. pallidipes, captured from

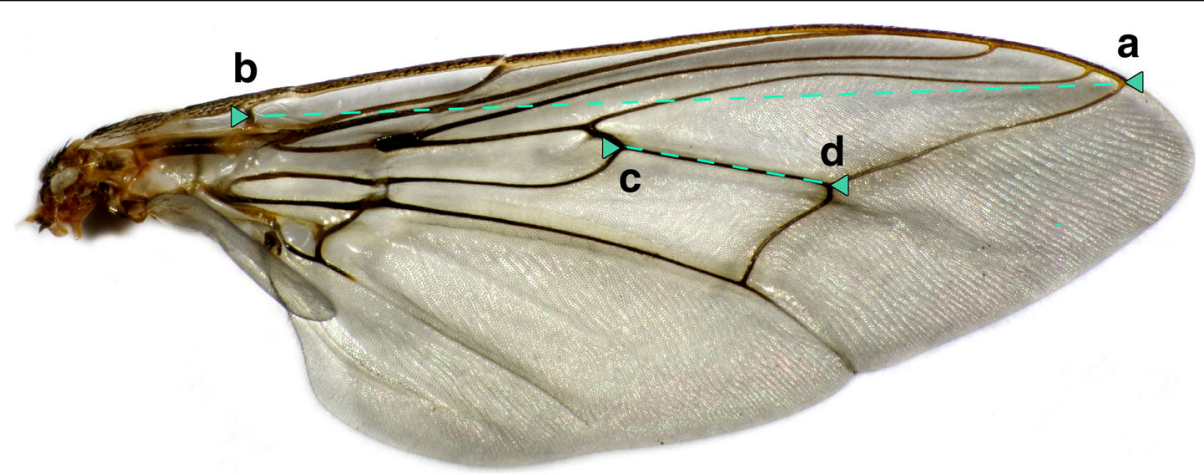

Fig. 1 Photograph of a tsetse wing. The right wing of a female G. $m$. morsitans showing the endpoints $\mathbf{a}$ and $\mathbf{b}$ used as a measure of wing length in present studies. The hatchet cell length used in classical studies is measured between points $\mathbf{c}$ and $\mathbf{d}$ 
artificial refuges and odour-baited traps, and subjected both to ovarian dissection and, thereafter, to nutritional analysis (see above).

Data were analysed using simple and multivariable linear regression, with error limits indicated by $95 \%$ confidence intervals. All data analyses were carried out using Microsoft Excel and Statacorp Stata v.14.2.

\section{Results}

\section{Measures of fly size}

Wing length vs centroid size (CS)

For the wings of 40 female G. pallidipes captured during January 1992, the mean monthly values of CS and wing length factor were highly correlated (Fig. 2).

\section{Wing length vs thoracic residual dry weight}

Regression analysis showed that thoracic residual dry weight (TRDW) was significantly correlated with our measure of wing length for female G. pallidipes captured using either artificial refuges or traps (Table 1). To ensure that all flies had completed the development of their thoracic musculature and exoskeletal structures, the analyses were restricted to flies that had produced their first larva.

\section{Gross effects of species and sex on wing length}

Table 2 shows the total numbers of flies sampled using traps, and the VET, during the study, and Table 3 shows the mean wing lengths among sub-samples of the captured flies of each sex and species in the pooled samples obtained from both baits in all seasons between January 1989 and December 1993. Since the principle interest of the overall study was in the age structure of the female population [31], the sample of flies examined was heavily biased in favour of females. Moreover, since the majority of flies were caught in traps - which capture G. pallidipes with a higher probability than G. m. morsitans - the sample was dominated by females of the first species.

Wings for females were, on average, 6.9\% longer than for males in G. pallidipes, and 8.6\% longer in G. m. morsitans (Student's t-test, $P<0.001$ in each case). The larger difference for the latter species means that there was little overlap in the distribution of wing lengths between male and female G. m. morsitans whereas the overlap was substantial for G. pallidipes (Table 3, 1\% and 99\% percentiles). For females, wings were $15.3 \%$ longer in G. pallidipes than in G. m. morsitans; for males the difference was $17.2 \%$. Since we will also discuss results for $G$. $f$. fuscipes, we note here that this species is approximately the same size as G. m. morsitans [32].

\section{Numbers of tsetse caught using the trap and VET Sex and species differences}

At almost all times of the year, and among all flies caught in both sampling devices, $<10 \%$ of male G. $m$.

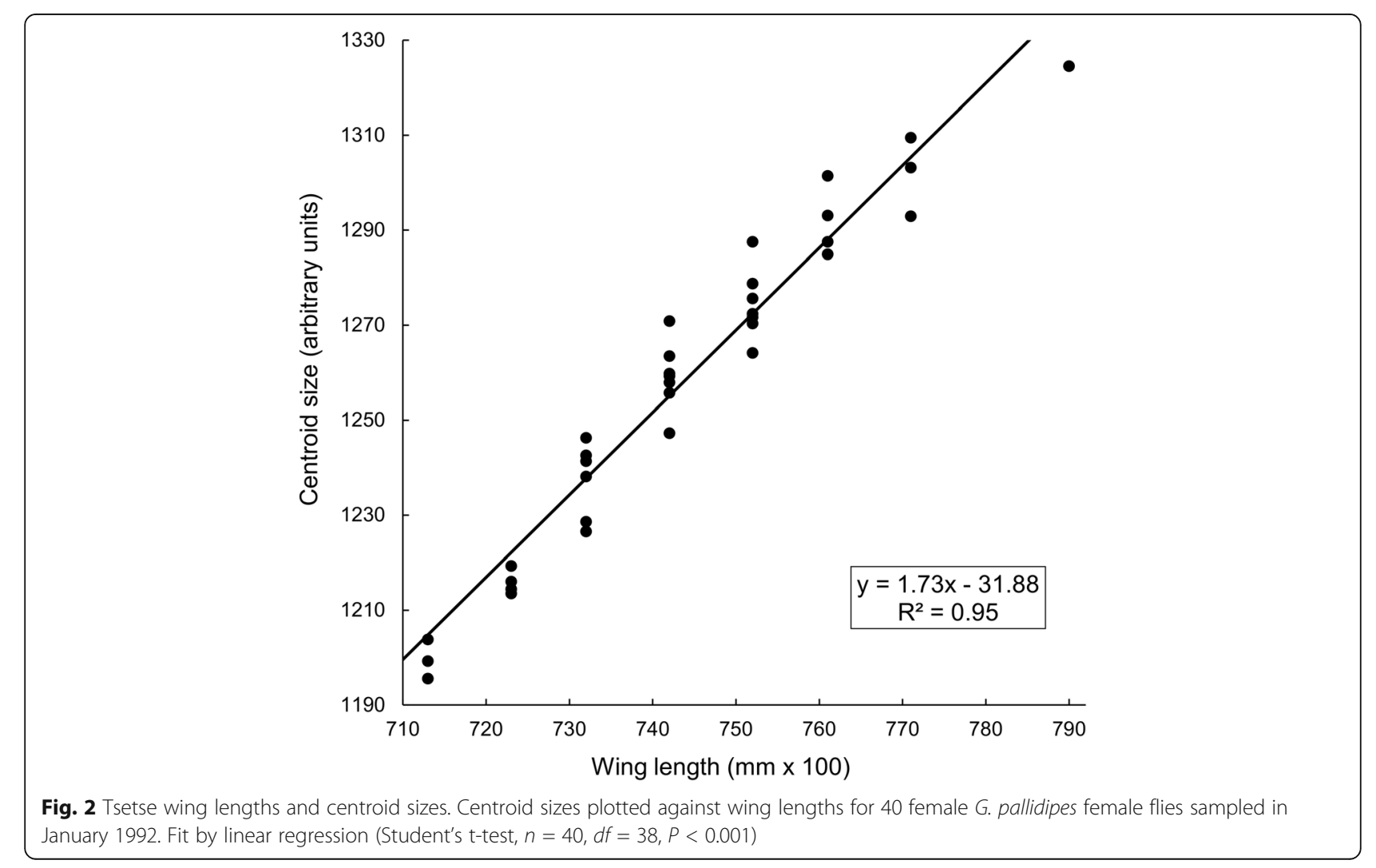


Table 1 Wing length and thoracic weight in tsetse. Linear regression of thoracic residual dry weight (TRDW) against wing length for female G. pallidipes captured in artificial refuges $(n=712)$ in October 1993 or odour-baited traps $(n=1352)$ in February 1994, at Rekomitjie

\begin{tabular}{lllllll}
\hline TRDW & & Coefficient & SE & Student's t statistic & $P$-value & 95\% Cl \\
\hline Wing length & Refuge & 1.75 & 0.10 & 16.92 & $<0.001$ & $<.55-1.96$ \\
& Trap & 1.63 & 0.06 & 25.20 & $<.001$ & $1.50-1.75$ \\
Constant & Refuge & -6.00 & 0.76 & -7.85 & $<0.001$ & $-7.50--4.50$ \\
& Trap & -4.76 & 0.47 & -10.11 & $<0.001$ & $-5.69--3.84$ \\
\hline
\end{tabular}

Abbreviations: $\mathrm{SE}$, standard error; $\mathrm{Cl}$, confidence interval

morsitans, the smallest flies, were captured in the stationary trap (Fig. 3). The relatively poor performance of the trap was less marked for female G. m. morsitans, but the percentage of these flies caught in the trap never exceeded $50 \%$. For G. pallidipes of both sexes, but particularly for females, the proportion captured in traps was greater than for G. m. morsitans. Within each sex and species, the proportion captured in traps varied with month, but the relative proportions always changed in the order indicated above. For both sexes of both species the relative proportions captured in the traps decreased as temperatures increased from September through November (Fig. 3).

\section{Differences between flies of different size, within a given sex and species group}

The results in Fig. 3 support the hypothesis that the larger the fly, i.e. female vs male and G. pallidipes vs G. $m$. morsitans, the greater is the chance the flies will be caught in a stationary trap rather than on the mobile VET. The percentage caught in traps varies with the month of the year but is generally $>10$ times higher for female G. pallidipes than for male G. m. morsitans. To determine whether the same principle applies to different size ranges of flies of the same sex and species, we

Table 2 Total catches of male and female G. m. morsitans and G. pallidipes sampled using traps and the VET at Rekomitjie between January 1989 and December 1993. The bottom row shows the percentage of the total catch for which the wing length was measured. For example, the wing length was measured in $41.6 \%$ of the 208,589 female G. pallidipes captured

\begin{tabular}{llllll}
\hline Method & \multicolumn{2}{l}{ G. m. morsitans } & & \multicolumn{2}{l}{ G. pallidipes } \\
\cline { 2 - 3 } & Males & Females & & Males & Females \\
\hline VET catches & 16,091 & 10,143 & & 14,284 & 19,773 \\
Sex ratio & & 0.63 & & 1.38 \\
Species ratio & & & 0.89 & 1.95 \\
Trap catches & 7230 & 10,575 & & 95,274 & 188,816 \\
Sex ratio & & 1.46 & & 1.98 \\
Species ratio & & & 13.18 & 17.85 \\
Total catches & 23,321 & 20,718 & & 109,558 & 208,589 \\
\% processed & 1.0 & 53.9 & 3.6 & 41.6 \\
\hline
\end{tabular}

compared the catch distribution among all female $G$. pallidipes with wings either larger or smaller than the mean length for all flies captured on the mobile and stationary systems. Given that wing lengths fluctuate markedly with time, it was necessary in doing these comparisons to restrict the analysis to situations where the flies processed were all captured on days when the trap and the VET were both run on the same afternoon, in the same location. Figure $4 \mathrm{a}$ shows that the mean wing length of flies in the upper half of the distribution was $4-5 \%$ greater than those in the lower half. Figure $4 \mathrm{~b}$, however, shows that while, in three months, the proportion of flies caught in traps was greater for larger flies, in the other five months the reverse was true; for most months the overlap of the $95 \%$ confidence intervals showed that the proportions did not differ significantly. There is thus no reason to believe that size difference among individuals within a given sex and species group will have much impact on availability.

\section{Seasonal effects on wing length}

Wing lengths for female G. pallidipes caught using the stationary trap and VET changed in a similar and highly predictable way with season (Fig. 5), falling steadily through the warm dry season, and increasing again once the rains started and temperatures fell. For most months, the complete overlap of $95 \%$ confidence intervals shows that there were no statistically significant differences between the wing lengths of flies captured using the two systems. Moreover, where there were significant differences, there was no consistency regarding the method that captured flies with longer wings. For example, whereas flies from the VET had significantly longer wings in March, April and December of 1989 (Fig. 5a), the reverse was the case in July and August 1991 (Fig. 5c). The pattern was the same for catches of female G. m. morsitans, but numbers were too low for a meaningful comparison of the month-by-month changes in catches from individual capture systems.

\section{Other factors affecting wing length}

The preceding results suggest that mean wing lengths vary little with the way that tsetse are sampled. There are, however, at least four complicating factors. First, the 
Table 3 Mean wing lengths of subsets of the captured flies

\begin{tabular}{|c|c|c|c|c|c|c|c|}
\hline \multirow[t]{2}{*}{ Species } & \multirow[t]{2}{*}{ Sex } & \multirow[t]{2}{*}{$n$} & \multirow[t]{2}{*}{ Percent } & \multirow{2}{*}{$\begin{array}{l}\text { Wing length } \\
(\mathrm{mm})\end{array}$} & \multirow[t]{2}{*}{$95 \% \mathrm{Cl}$} & \multicolumn{2}{|c|}{ Percentiles } \\
\hline & & & & & & $1 \%$ & $99 \%$ \\
\hline \multirow[t]{2}{*}{ G. m. morsitans } & Males & 238 & 1.0 & 5.830 & $5.800-5.859$ & 5.47 & 6.65 \\
\hline & Females & 11,162 & 53.9 & 6.334 & $6.329-6.338$ & 5.75 & 6.83 \\
\hline \multirow[t]{2}{*}{ G. pallidipes } & Males & 3954 & 3.6 & 6.830 & $6.821-6.838$ & 6.14 & 7.48 \\
\hline & Females & 86,675 & 41.6 & 7.303 & $7.302-7.305$ & 6.68 & 7.81 \\
\hline
\end{tabular}

Abbreviations: $n$, sample size on which the means were calculated; $\mathrm{Cl}$, confidence interval

Note: $1 \%$ of the $n$-values lie below/above the $1 \% / 99 \%$ percentiles

age structures of samples vary significantly depending on the sampling method [31]. Secondly, the relationship between fly size and fly age also changes with season $[26,27]$ : in estimating the effect of capture method on wing length we should, therefore, adjust for fly age and season. Thirdly, it also appears that wing lengths change from year to year and we need to adjust for these changes. For example, the highest wing lengths in July 1992 and 1993 were $7.38 \mathrm{~mm}$ (95\% CI: 7.37-7.39 mm) and $7.54 \mathrm{~mm}$ (95\% CI: 7.53-7.55 mm), respectively, with no overlap of the $95 \%$ confidence intervals. Similarly, the lowest mean wing lengths in 1992, $7.05 \mathrm{~mm}$ (95\% CI: 7.03-7.70 mm), occurred in November and were significantly shorter than the shortest lengths in 1993, 7.16 $\mathrm{mm}$ (95\% CI: 7.15-7.18 mm) which occurred in December (Student's t-test, $P<0.001$ for all of the differences).
Finally, there were significant interactions between some of the above-named variables.

\section{Wing length as a function of ovarian age: interaction with month of capture}

Over the period 1959-2015, 95\% of the average annual rainfall at Rekomitjie fell in the months November-March, with $73 \%$ in December-February. With the onset of the main rains in December, the size of young flies began to increase, a trend that accelerated in January and February (Fig. 6a, c). Thereafter, from March until July, there was little change in the wing lengths of flies in ovarian category 0 (Fig. 6a, b). The net result of these changes was that wing length decreased more steeply with increasing ovarian age with each successive month between December and February. This trend slowed in March-April,

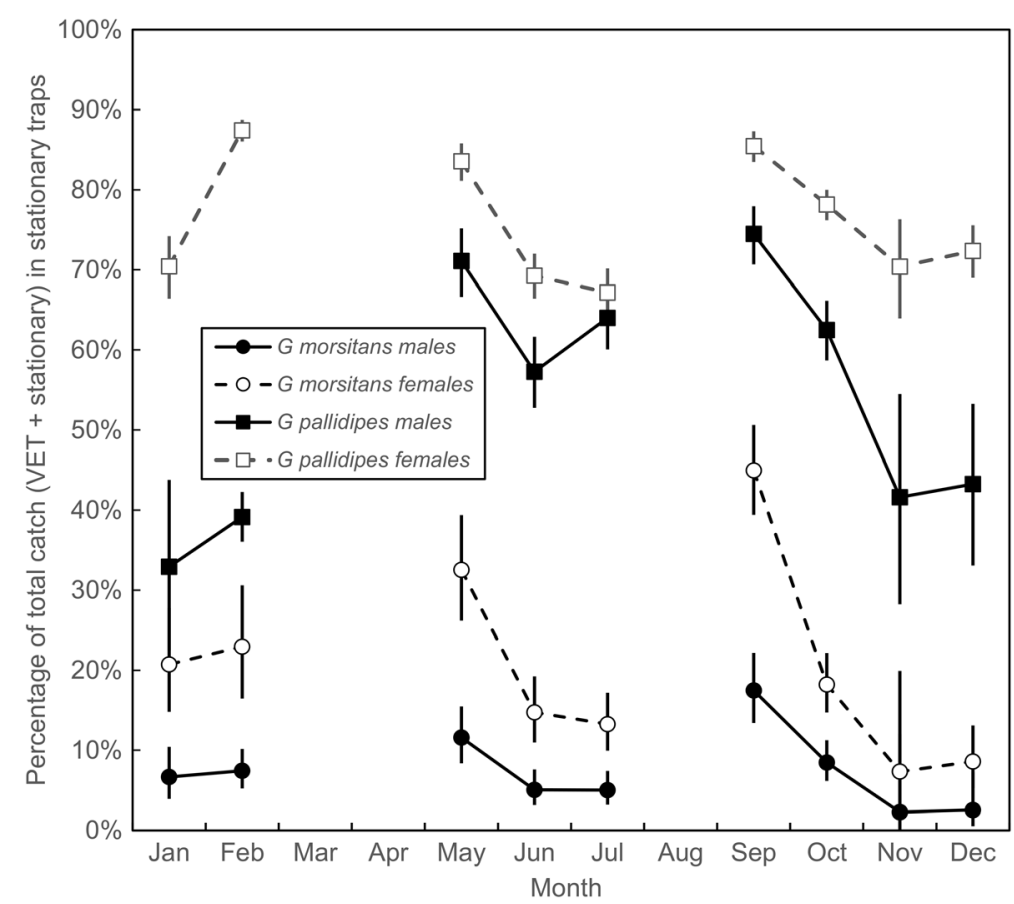

Fig. 3 Catches of tsetse from trap and VET. Male and female G. m. morsitans and G. pallidipes caught using stationary traps and the VET. For each month, stationary-trap catches are expressed as a percentage of the total captured by the stationary trap and the VET for that particular sexspecies combination. Both devices were run in the same area at Rekomitjie during approximately the last two hours of daylight on 59 days between January and December 1992 

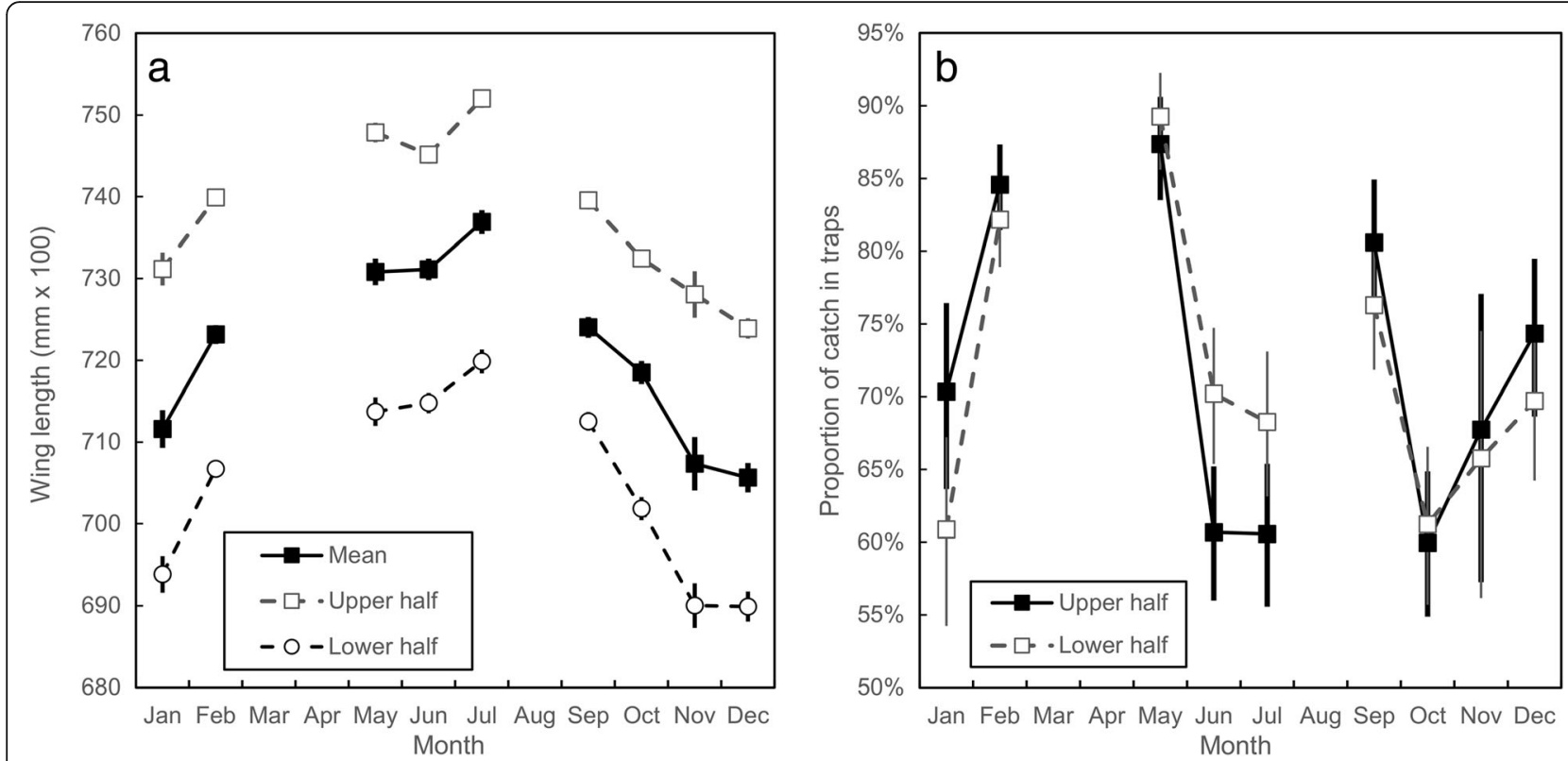

Fig. 4 Wing lengths and numbers of female G. pallidipes captured using a stationary trap or VET. a Mean wing length for female G. pallidipes caught in the trap or VET at Rekomitjie, during approximately the last two hours of daylight on 59 days between January and December 1992, when both sampling systems were used on the same day in the same area. Catches were pooled by month. Means and confidence intervals are calculated for the pooled data and for the upper and lower halves of the distributions. b Monthly estimates for the numbers of female G. pallidipes caught in odour-baited traps as a proportion of the total captured using a stationary trap and the VET

when there was no further increase in the size of young flies: between May and July wing length was almost independent of ovarian category (Fig. 6b). In August, when temperatures started to increase, the wing lengths of young flies began to decrease: this trend accelerated through November, with the result that wing length then increased ever more steeply with increasing ovarian age, with each successive month between August and November (Fig. 6c).

\section{Multivariable analysis of factors affecting wing length in females of both species}

Given the foregoing results, we performed regression analyses with wing length as the dependent variable and with the following independent variables: ovarian category, capture month, capture year and capture method. As before, the data were restricted for this analysis to the period when both traps and the VET were used to sample flies (January 1989 to December 1993). Only about $5 \%$ of the data available were for males, for which there was no accurate measure of age; accordingly, we only analysed the data for female tsetse. We used likelihood ratios to test for statistically significant interactions between independent variables, comparing results of runs where interactions were, or were not, included. For G. pallidipes, there were statistically significant interactions between ovarian category and each of capture month, year and method. For G. m. morsitans there was a significant interaction between ovarian category and capture month, but not with capture year or method. The complete results of the full model analyses are shown in Additional file 1: Table S1; results for the runs where only the main effects were included are shown in Table 4 .

The results of the full multivariable analysis were used to predict the wing lengths for each ovarian category of flies captured in traps in each month across the 60 months of the study (Fig. 7). The sizes for flies caught using the VET were similarly calculated using the coefficients in the regression equation for method, and the interaction between capture month and method. Results for all ovarian categories were closely similar in showing that, in absolute terms, wing length hardly varied with capture method. For female G. pallidipes (Table 4), the average wing length of flies caught on the VET was just $0.0173 \mathrm{~mm}$ shorter than for flies caught in the trap, which is only $0.24 \%$ of the mean length of $7.078 \mathrm{~mm}$. For G. m. morsitans the difference was $0.0297 \mathrm{~mm}$, which is $0.48 \%$ of $6.183 \mathrm{~mm}$ (Table 4). These apparent variations due to capture method are much less than the total background variation. For example, with G. pallidipes the coefficient for the effect of month of capture varied between 32.77 in July and -1.53 in December, a difference of 34.30. This was 19.8 times greater that the bait effect of 1.73 (Table 4). For G. m. morsitans the shift between the same months was $27.85-(-5.85)=33.70$, 11.3 times greater than the bait effect of 2.97 . 

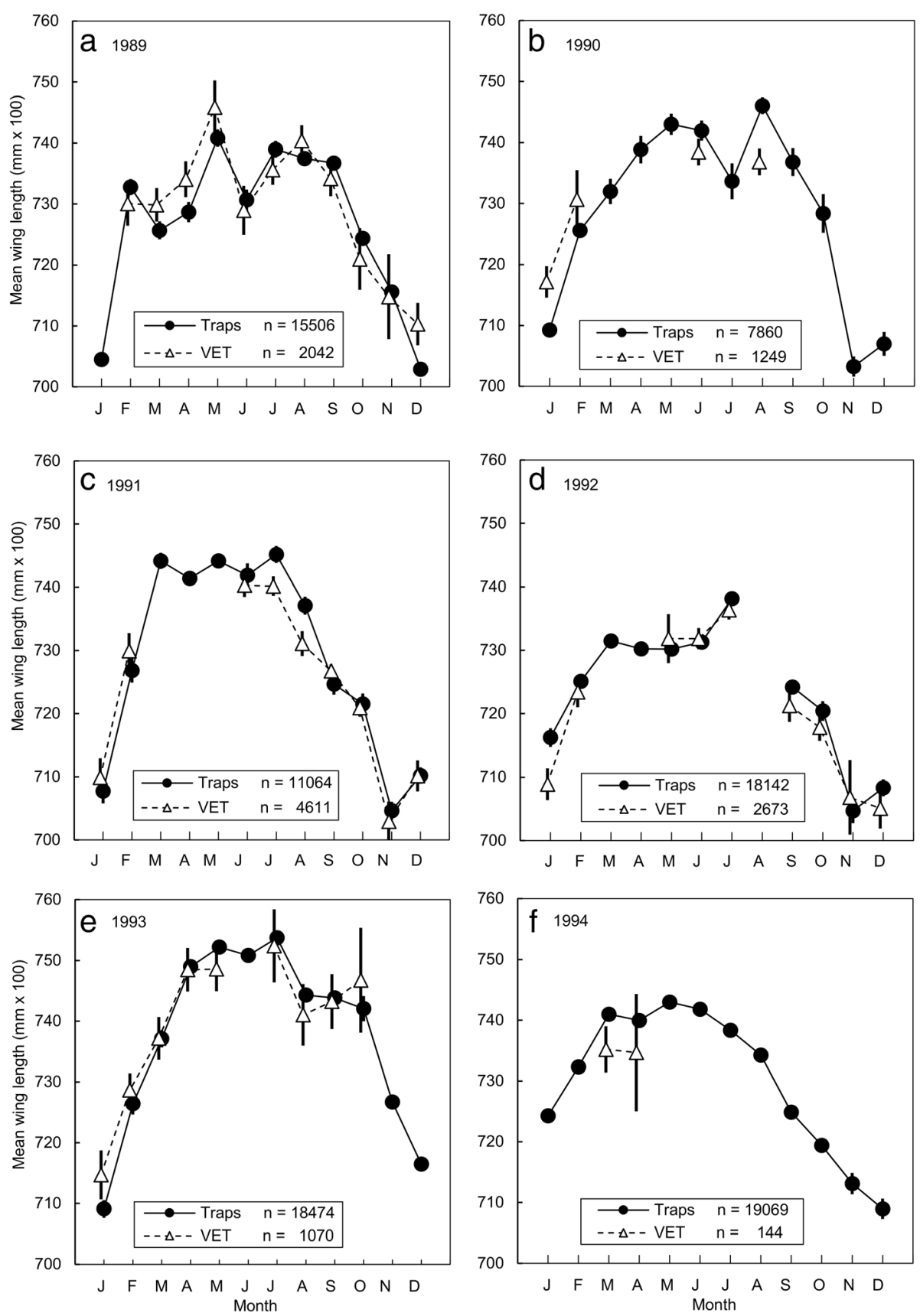

Fig. 5 Wing length as a function of month and method of capture, for the following years: a 1989; b 1990; c 1991; d 1992; e 1993; f 1994. Monthly means of wing lengths for female G. pallidipes captured using odour-baited traps or a vehicle-mounted electric net (VET). Data for each capture method pooled over all sampling sites at Rekomitjie

\section{Discussion}

Our results (Tables 2, 3; Fig. 3) confirm earlier indications that the availability of tsetse to stationary baits, as compared to mobile ones, is much greater for the taxa of larger wing size, i.e. greater for G. pallidipes than for G. m. morsitans, and greater for females than for males. The results provide little support, however, for the idea that larger individuals within a taxon are selectively attracted to stationary baits: subgroups of female G. pallidipes that were either larger or smaller than average showed no consistent size-related biases in such behaviour (Fig. 4). While multivariable analysis of the effects of month, year, fly age and capture method showed a statistically significant effect of bait type on fly size, the absolute magnitude of this effect was very small (Table 4) and much less than the total background variation. 

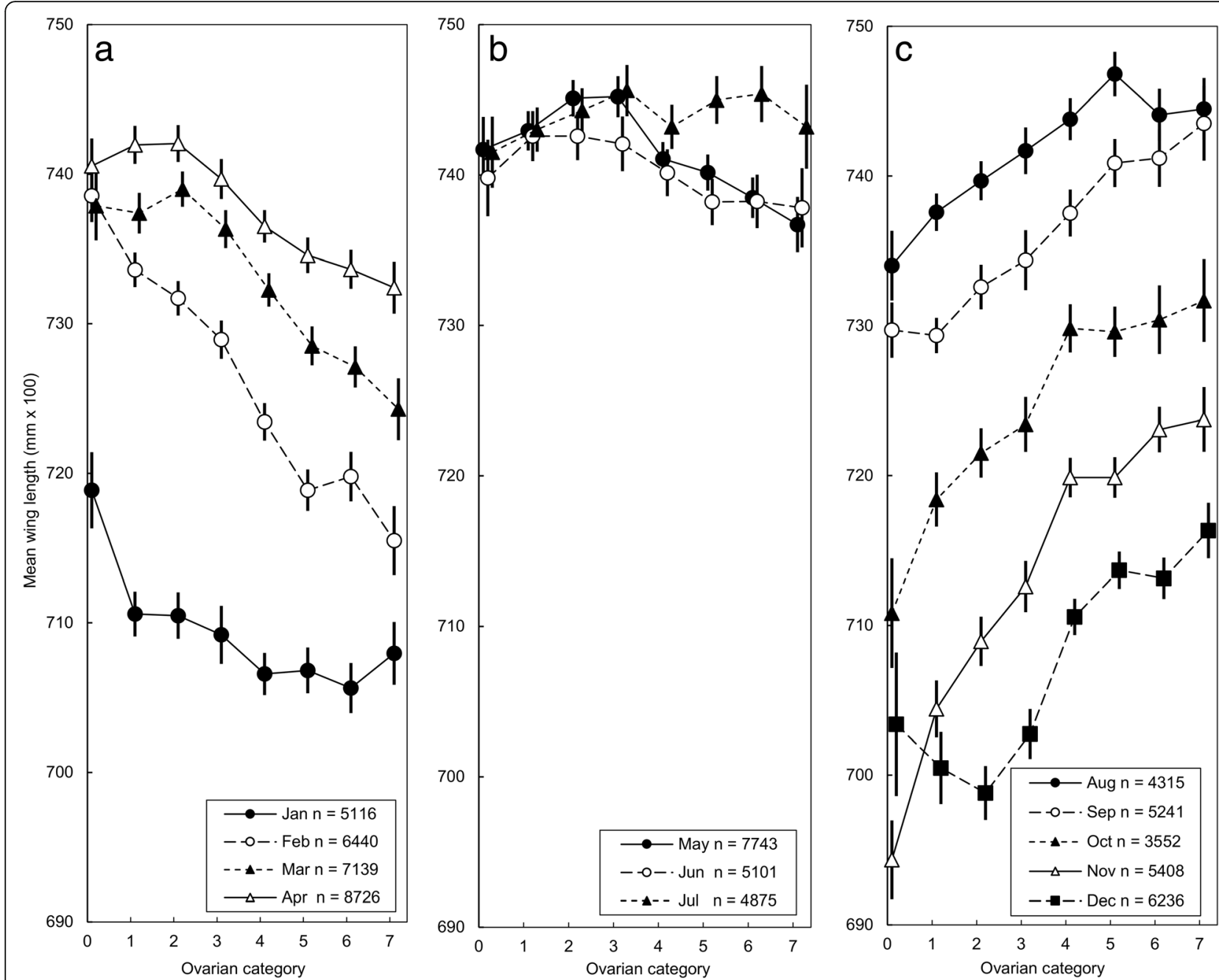

Fig. 6 Tsetse wing lengths as a function of age and month of capture: a January - April; b May - July; c August - December. Mean wing lengths of female G. pallidipes as a function of ovarian age and month of capture. Flies caught in odour-baited traps at Rekomitjie from January 1989 to December 1993

For example, with G. pallidipes the average seasonal effect (Table 4) was on average 20 times greater that the apparent bait effect, and for G. m. morsitans it was 11 times greater. Moreover, the mean size variation between the two species was 30-52 times the bait effect.

The present indications of wide background variations in fly size associated with season and year are consistent with published evidence [33-37]. There is also evidence that tsetse caught in localities just one or a few kilometres apart can differ in mean size and in the seasonal pattern of size variations [38, 39]. To this variability might be added the expectation that changes in host availability in isolated populations would affect the nutritional state of female tsetse and hence alter the size of offspring. Given the plethora of factors liable to affect the mean size of tsetse, we must thus join Glasgow \& Bursell [36] in warning against accepting what might appear the simplest or most appealing interpretation of size differences between flies collected at different times and places, especially if the samples do not contain many tsetse, and are not obtained with systematic and comprehensive attention to what are likely to be the main sources of background variation.

Our results for G. pallidipes and G. m. morsitans thus provide no support for the idea that campaigns using (stationary) targets could cause any meaningful shift towards tsetse that are relatively small, immobile and unavailable to targets [11]. The ability to eradicate these species thus seems unimpeded by any size-bias in the availability of tsetse to these baits. This accords with the results of various successful target-based operations in Zimbabwe [40-42] and with the calculations of the probability of extinction resulting from such operations [43]. 
Table 4 Multivariable analysis of the effects of ovarian age, month, year and method of capture on the wing length of female $G$. pallidipes ( $\left.n=80,219 ; r^{2}=0.26\right)$ and G. m. morsitans $\left(n=7764 ; r^{2}=0.24\right)$ captured in the field at Rekomitjie between January 1989 and December 1993

\begin{tabular}{|c|c|c|c|c|c|c|}
\hline & \multicolumn{3}{|l|}{ G. pallidipes } & \multicolumn{3}{|c|}{ G. m. morsitans } \\
\hline & \multirow[t]{2}{*}{ Coefficient } & \multicolumn{2}{|l|}{$95 \% \mathrm{CL}$} & \multirow[t]{2}{*}{ Coefficient } & \multicolumn{2}{|l|}{$95 \% \mathrm{CL}$} \\
\hline & & Lower & Upper & & Lower & Upper \\
\hline \multicolumn{7}{|l|}{ Ovarian age } \\
\hline 1 & -1.21 & -1.88 & $-0.54^{* * *}$ & -2.01 & -3.88 & $-0.13^{*}$ \\
\hline 2 & 0.22 & -0.44 & 0.88 & -2.45 & -4.37 & $-0.54^{*}$ \\
\hline 3 & 0.35 & -0.33 & 1.04 & -1.26 & -3.26 & 0.74 \\
\hline 4 & 0.22 & -0.43 & 0.87 & 0.94 & -0.91 & 2.80 \\
\hline 5 & 0.05 & -0.61 & 0.72 & -1.72 & -3.65 & 0.21 \\
\hline 6 & -0.18 & -0.89 & 0.52 & -0.97 & -3.09 & 1.14 \\
\hline 7 & -0.22 & -1.03 & 0.60 & -0.61 & -3.52 & 2.31 \\
\hline \multicolumn{7}{|l|}{ Year } \\
\hline 90 & 1.67 & 1.10 & $2.25^{* * *}$ & 2.47 & 0.33 & $4.60^{*}$ \\
\hline 91 & 2.27 & 1.79 & $2.76^{* * *}$ & 5.08 & 3.46 & $6.70^{* * *}$ \\
\hline 92 & -2.85 & -3.31 & $-2.40^{* * *}$ & -2.27 & -4.10 & $-0.43^{*}$ \\
\hline 93 & 11.73 & 11.28 & $12.18^{* * *}$ & 8.84 & 6.99 & $10.69^{* * *}$ \\
\hline \multicolumn{7}{|l|}{ Month } \\
\hline February & 18.45 & 17.71 & $19.19^{* * *}$ & 9.42 & 7.08 & $11.77^{* * *}$ \\
\hline March & 24.88 & 24.14 & $25.63^{* * *}$ & 17.89 & 14.97 & $20.82^{* * *}$ \\
\hline April & 27.35 & 26.63 & $28.06^{* * *}$ & 24.97 & 21.83 & $28.12^{* * *}$ \\
\hline May & 30.86 & 30.12 & $31.59^{* * *}$ & 22.70 & 19.55 & $25.85^{* * *}$ \\
\hline June & 29.22 & 28.46 & $29.98^{* * *}$ & 23.09 & 20.83 & $25.34^{* * *}$ \\
\hline July & 32.77 & 32.00 & $33.54^{* * *}$ & 27.85 & 25.60 & $30.10^{* * *}$ \\
\hline August & 27.78 & 26.97 & $28.58^{* * *}$ & 23.04 & 20.63 & $25.45^{* * *}$ \\
\hline September & 22.50 & 21.73 & $23.26^{* * *}$ & 21.03 & 18.73 & $23.32^{* * *}$ \\
\hline October & 15.68 & 14.84 & $16.51^{* * *}$ & 13.04 & 10.58 & $15.51^{* * *}$ \\
\hline November & 3.24 & 2.45 & $4.03^{* * *}$ & -3.27 & -5.86 & $-0.69^{*}$ \\
\hline December & -1.53 & -2.29 & $-0.78^{* * *}$ & -5.85 & -8.25 & $-3.49 * * *$ \\
\hline \multicolumn{7}{|l|}{ Method } \\
\hline VET & -1.73 & -2.21 & $-1.26^{* * *}$ & -2.97 & -4.23 & $-1.73^{* * *}$ \\
\hline Constant & 707.8 & 706.9 & 708.6 & 618.3 & 615.6 & 621.1 \\
\hline
\end{tabular}

${ }^{*} P<0.05,{ }^{* * *} P<0.001$ (Student's t-test)

Abbreviation: $\mathrm{CL}, 95 \%$ confidence interval limits

Targets have, however, often been used in areas where there was no intention or expectation of eliminating the flies, especially in those very common situations where invasion can occur from neighbouring untreated areas (e.g. $[12,41])$, as would be the case, for example, if odour-baited targets were deployed in the neighbourhood of Rekomitjie Research Station. In such places, no control method of any sort can eliminate the flies unless invasion is specifically prevented. Targets can be particularly useful in providing an invasion barrier [17, 44], and consequently they are often used in association with other control techniques [45].
The situation might be different for G. f. fuscipes, which does not use odour in host location in the manner of G. m. morsitans and, particularly, G. pallidipes. The proportion of the population trapped per target is thus much smaller for G. f. fuscipes. The target density required to achieve a desired rate of population reduction is thus much higher for G. $f$. fuscipes than for the Zimbabwe species. This implies that G. f. fuscipes need not travel so far as G. pallidipes in order to find a target, so reducing any selection against large and relatively mobile individuals. Various considerations suggest, anyway, that more data need to be produced before one could 

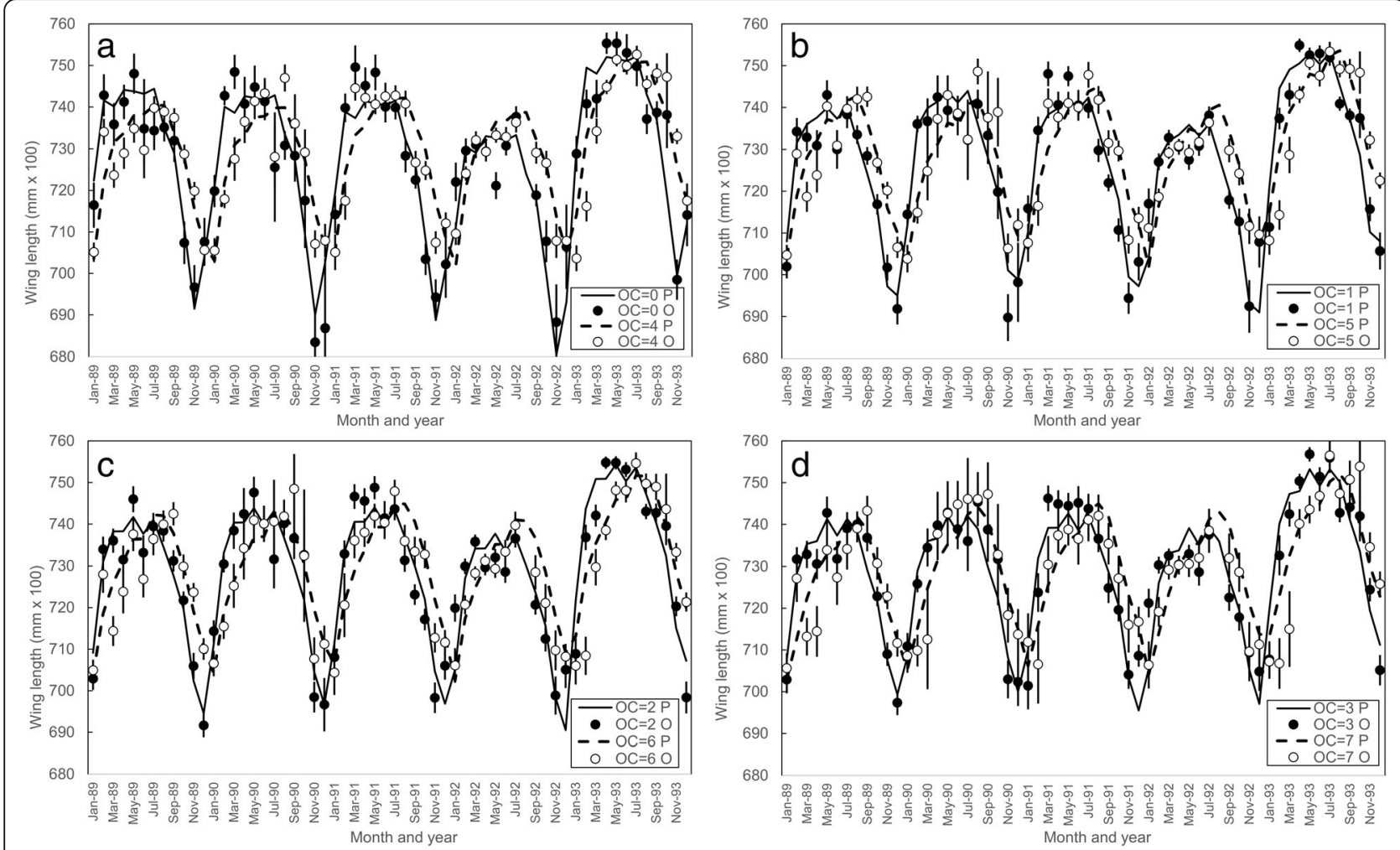

Fig. 7 Mean wing lengths for flies in different ovarian age categories $(O C)$ caught using odour-baited traps or the VET: a OC = 0 and 4 ; $\mathbf{b}$ OC $=1$ and 5; $\mathbf{c} O C=2$ and $6 ; \mathbf{d} O C=3$ and 7. Mean wing lengths for flies in different ovarian age categories (C) caught using odour-baited traps or the VET. Observed values $(\mathrm{O})$ are the monthly means for all flies captured using the two sampling systems. Predicted values (P) are calculated using the results in Additional file 1: Table S1. Rekomitjie; January 1989 to December 1993

unequivocally attribute the size changes recorded in the recent study on G. f. fuscipes in Kenya to the use of targets in the control campaign [11]. First, the possibility could not be excluded that increases in fly numbers following control operations in the study area were due to invasion from neighbouring areas [11]. If that were the case, it would be unclear whether there was a real change in the size of tsetse in the original population within the operational area, and how great that change might be, and what might have caused it. Secondly, the study involved comparing samples, captured using only stationary traps, collected over just three days in a two-month period with samples obtained during many days in a 19-month period, four to five years previously, with no sampling in-between.

Even if the results are accepted at face value, it appears that the 19-months of suppression due to targets deployed on Big Chamaunga Island reduced the mean size of female tsetse on the island by only about 1\% [11]. This very small effect appears compatible with present evidence that, within a taxon, the mean sizes of tsetse available to mobile and stationary baits differ minimally compared to gross variations associated with sex, species, and environmental factors. It is also consistent with the suggestion that the size-associated responsiveness of tsetse is part of the innate behavioural repertoire of all flies of each taxon, which takes account of the average size of the tsetse within it [5]. Finally, it is consistent with Brady's [8] demonstration that spontaneous activity is innate, and increases in intensity as the hunger cycle develops. The increased activity enhances the probability of locating stationary, as well as mobile, baits. Flies that systematically avoided behaviour that could lead to finding stationary baits would be more likely to starve, thereby reducing the likelihood of effective genetic selection in favour of such avoidance.

\section{Possible limitations of our work}

Given that the flies used in our analysis were sampled up to 30 years ago, the question may arise as to the usefulness of our results in modern contexts. As indicated in the Methods section, Rekomitjie is situated within a very large protected area and there have been no major changes to the environment around Rekomitjie due to human settlement or agricultural activity. We cannot exclude the possibility of environmental changes due to climate and weather changes over the past few decades. Such changes would, however, only be important to our analysis if it affected the relative sizes of tsetse caught 
using mobile and stationary baits. Results from our study suggest that such effects are unlikely. Thus, as with most field situations in most environments, there are major, annual, cyclical changes in the weather at Rekomitjie, and these are associated with changes in vegetation and in animal abundance and distribution. During the course of the study there were also major differences between years in the temperatures measured at the station. As indicated by our results (Fig. 4), these changes are indeed associated with changes in the size of tsetse captured at different times of the year. The important point, however, is that the sizes of flies taken from our mobile and stationary sampling systems changed in very similar ways with time. There seems no reason to suggest, therefore, that any long-term environmental changes will have affected the conclusions of our study.

\section{Conclusions}

Stationary odour-baited traps show a very small absolute bias in favour of larger flies within a taxon. There seems no reason to believe that stationary odour-baited targets are incapable of eliminating tsetse from suitably isolated situations, nor that they will affect materially the feeding behaviour of tsetse, nor that catches from stationary sampling devices will give seriously misleading indices of changes in tsetse population density.

\section{Additional file}

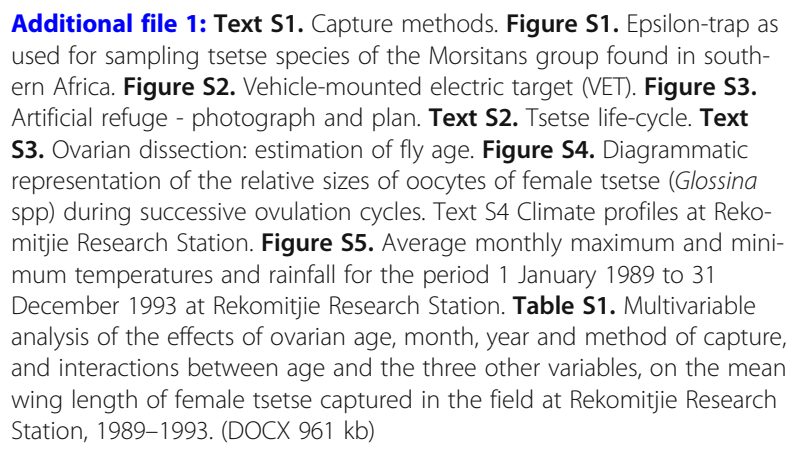

\section{Abbreviations}

Cl: Confidence interval; CS: Centroid size; SACEMA: Department of Science and Technology/National Research Foundation Centre of Excellence in Epidemiological Modelling and Analysis; TRDW: Thoracic residual dry weight; UK: United Kingdom; UNESCO: United Nations Educational, Scientific and Cultural Organization; VET: Vehicle-mounted electric target

\section{Acknowledgements}

Mr William Shereni, Director of the Trypanosomiasis Control Department, Government of Zimbabwe gave us continued support. All experimental work was carried out at Rekomitjie Research Station in the Zambezi Valley. We thank the tsetse capture team ably led by Mr Pio Chimanga and the expert and dedicated ovarian dissection team.

\section{Funding}

SACEMA receives core funding from the Department of Science and Technology, Government of South Africa. This project received financial support from the DFID Animal Health Programme (project nos. R7539 and R7987), and the Biotechnology and Biological Sciences Research Council (BBSRC) under the programme on Zoonoses and Emerging Livestock Systems (ZELS, grant no. BB/ L019035/1) and a Responsive Mode grant (BB/P006159/1). SE was supported by a Royal Society Dorothy Hodgkin Fellowship. The contents of this publication are the sole responsibility of the authors and do not necessarily reflect the views of any funding agency.

\section{Availability of data and materials}

Data supporting the conclusions of this article are included within the article and its additional file. The datasets analysed during the present study are available from the corresponding author upon reasonable request.

\section{Authors' contributions}

JWH carried out data collection, collation, cleaning and analysis. CvS oversaw statistical analysis. LRH took the photographs of the tsetse wing. All authors contributed to the writing of the manuscript, and read and approved the final version.

Ethics approval and consent to participate

Not applicable.

Consent for publication

Not applicable.

Competing interests

The authors declare that they have no competing interests.

\section{Publisher's Note}

Springer Nature remains neutral with regard to jurisdictional claims in published maps and institutional affiliations.

\section{Author details}

${ }^{1}$ SACEMA, University of Stellenbosch, Stellenbosch, South Africa. ${ }^{2}$ School of Biological Sciences, University of Bristol, Bristol, UK. ${ }^{3}$ Liverpool School of Tropical Medicine, Liverpool, UK. ${ }^{4}$ London School of Hygiene and Tropical Medicine, London, UK. ${ }^{5}$ Natural Resources Institute, University of Greenwich, London, UK.

Received: 26 April 2018 Accepted: 19 December 2018

Published online: 11 January 2019

\section{References}

1. Leak S. Tsetse Biology and Ecology. Wallingford, UK: CABI Publishing; 1998.

2. Maudlin I, Holmes P, Miles P. The Trypanosomiases. Wallingford, UK: CABI Publishing; 2004.

3. Fevre EM, Picozzi K, Jannin J, Welburn SC, Maudlin I. Human African trypanosomiasis: epidemiology and control. Adv Parasitol. 2006;61:167-221.

4. Welburn SC, Molyneux DH, Maudlin I. Beyond tsetse - implications for research and control of human African trypanosomiasis epidemics. Trends Parasitol. 2016;32:230-41.

5. Vale GA, Hargrove JW, Solano P, Courtin F, Rayaisse J-B, Lehane MJ, et al. Explaining the host-finding behavior of blood-sucking insects: computerized simulation of the effects of habitat geometry on tsetse fly movement. PLoS Negl Trop Dis. 2014;8:e2901.

6. Bursell E, Billing K, Hargrove J, McCabe C, Slack E. Metabolism of the bloodmeal in tsetse flies (a review). Acta Trop. 1974;31:297-30.

7. Brady J. The visual responsiveness of the tsetse fly Glossina morsitans Westw. (Glossinidae) to moving objects: the effects of hunger, sex, host odour and stimulus characteristics. Bull Entomol Res. 1972;62:257-79.

8. Brady J. Spontaneous, circadian components of tsetse fly activity. J Insect Physiol. 1972;18:471-84.

9. Hargrove J, Packer M. Nutritional states of male tsetse flies (Glossina spp.) (Diptera: Glossinidae) caught in odour-baited traps and artificial refuges: models for feeding and digestion. Bull Entomol Res. 1993;83:29-46.

10. Hargrove J, Williams B. A cost-benefit analysis of feeding in female tsetse. Med Vet Entomol. 1995;9:109-19.

11. Mbewe NJ, Saini RK, Torto B, Irungu J, Yusuf AA, Pirk C. Effects of vector control on the population structure of tsetse (Glossina fuscipes fuscipes) in western Kenya. Acta Trop. 2018;179:1-9. 
12. Tirados I, Esterhuizen J, Kovacic V, Mangwiro TNC, Vale GA, Hastings I, et al. Tsetse control and Gambian sleeping sickness; implications for control strategy. PLoS Negl Trop Dis. 2015;9:e0003822.

13. Meyer A, Holt HR, Selby R, Guitian J. Past and ongoing tsetse and animal trypanosomiasis control operations in five African countries: a systematic review. PLoS Negl Trop Dis. 2016;10:e0005247.

14. Vreysen MJB, Seck MT, Sall B, Bouyer J. Tsetse flies: their biology and control using area-wide integrated pest management approaches. J Invertebr Pathol. 2013;112(Suppl.):S15-25.

15. Hansen MC, Potapov PV, Moore R, Hancher M, Turubanova SA, Tyukavina A, et al. High-resolution global maps of 21st-century forest cover change. Science. 2013;342:850-3.

16. Dunham KM, Mackie CS, Nyaguse G. Aerial survey of elephants and other large herbivores in the Zambezi Valley (Zimbabwe): 2014. In: Great Elephant Census. Seattle, WA, USA: Vulcan Inc.; 2015. p. 118.

17. Muzari MO, Hargrove JW. The design of target barriers for tsetse flies, Glossina spp. (Diptera: Glossinidae). Bull Entomol Res. 1996;86:579-83.

18. Vale GA. New field methods for studying the responses of tsetse flies (Diptera, Glossinidae) to hosts. Bull Entomol Res. 1974;64:199-208.

19. Hargrove J. Reproductive abnormalities in tsetse flies in Zimbabwe. Entomol Exp Appl. 1999;92:89-99.

20. Saunders BDS. The ovulation cycle in Glossina morsitans Westwood (Diptera: Muscidae) and a possible method of age determination for female tsetse flies by the examination of their ovaries. Trans R Entomol Soc London. 1960; 112:221-38.

21. Saunders D. Age determination for female tsetse flies and the age compositions of samples of Glossina pallidipes Aust., G. palpalis fuscipes Newst. and G. brevipalpis Newst. Bull Entomol Res. 1962;53:579-96.

22. Challier A. Amélioration de la méthode de détermination de l'âge physiologique des glossines. Études faites sur Glossina palpalis palpalis Vanderplank. Bull la Société Pathol Exot. 1949;58:250-9.

23. Challier A. Ecologie de Glossina palpalis gambiensis Vanderplank, 1949 (Diptera-Muscidae) en savanne d'Afrique Occidentale. Mémoire ORSTOM. 1949;64:274.

24. Hargrove JW. Age-specific changes in sperm levels among female tsetse (Glossina spp.) with a model for the time course of insemination. Physiol Entomol. 2012;37:278-90.

25. Vale G. Artificial refuges for tsetse flies (Glossina spp.). Bull Entomol Res. 1971;61:331-50.

26. Hargrove J. Nutritional levels of female tsetse Glossina pallidipes from artificial refuges. Med Vet Entomol. 1999;13:150-64.

27. Hargrove J. Lifetime changes in the nutritional characteristics of female tsetse Glossina pallidipes caught in odour-baited traps. Med Vet Entomol. 1999:13:165-76.

28. Jackson CHN. An artificially isolated generation of tsetse flies (Diptera). Bull Entomol Res. 1946;37:291-9.

29. Dujardin J-P, Slice D. Contributions of morphometrics to medical entomology. In: Turkington C, Ashby B, editors. The Encyclopedia of Infectious Diseases: Modern Methodologies. New York: Infobase Publishing; 2007. p. 415.

30. Bursell E. The measurement of size in tsetse flies (Glossina). Bull Entomol Res. 1960;51:33-7.

31. Hargrove J, Ackley S. Mortality estimates from ovarian age distributions of the tsetse fly Glossina pallidipes Austen sampled in Zimbabwe suggest the need for new analytical approaches. Bull Entomol Res. 2015;105:294-304.

32. Glasgow J. The genus Glossina: Introduction. In: Mulligan HW, editor. The African Trypanosomiases. London: George Allen and Unwin; 1970. p. 950.

33. Jackson C. Seasonal variation in the mean size of tsetse flies. Bull Entomol Res. 1953:43:703-6.

34. Glasgow JP. Selection for size in tsetse flies. J Anim Ecol. 1961;30:87-94.

35. Glasgow JP. Seasonal variation in size and colour, and daily changes in the distribution of Glossina pallidipes Aust. in the South Busoga Forest, Uganda. J Anim Ecol. 1963;31:647-66.

36. Glasgow JP, Bursell E. Seasonal variations in the fat content and size of Glossina swynnertoni Austen. Bull Entomol Res. 1961;51:705-13.

37. Dransfield RD, Brightwell R, Kiilu J, Chaudhury MF. Size and mortality rates of Glossina pallidipes in the semi-arid zone of southwestern Kenya. Med Vet Entomol. 1989;3:83-95.

38. Bursell $E$. The nutritional state of tsetse flies from different vegetation types in Rhodesia. Bull Entomol Res. 1966;57:171-80.
39. Vale GA, Cumming DHM. The effects of selective elimination of hosts on a population of tsetse flies (Glossina morsitans morsitans Westwood (Diptera, Glossinidae)). Bull Entomol Res. 1976;66:713-29.

40. Vale GA, Hargrove JW, Cockbill GF, Phelps RJ. Field trials of baits to control populations of Glossina morsitans morsitans Westwood and G. pallidipes Austen (Diptera: Glossinidae). Bull Entomol Res. 1986;76:179-93.

41. Vale G, Lovemore D, Flint S, Cockbill G. Odour-baited targets to control tsetse flies, Glossina spp. (Diptera: Glossinidae) in Zimbabwe. Bull Entomol Res. 1988;78:31-49.

42. Hargrove JW. Tsetse eradication: sufficiency, necessity and desirability. In: Research report, DFID Animal Health Programme, Centre for Tropical Veterinary Medicine. Edinburgh, UK: University of Edinburgh; 2003.

43. Hargrove J. Extinction probabilities and times to extinction for populations of tsetse flies Glossina spp. (Diptera: Glossinidae) subjected to various control measures. Bull Entomol Res. 2005;95:13-21.

44. Hargrove J. Target barriers for tsetse flies (Glossina spp.) (Diptera: Glossinidae): quick estimates of optimal target densities and barrier widths. Bull Entomol Res. 1993;83:197.

45. Kgori PM, Modo S, Torr SJ. The use of aerial spraying to eliminate tsetse from the Okavango Delta of Botswana. Acta Trop. 2006;99:184-99.
Ready to submit your research? Choose BMC and benefit from:

- fast, convenient online submission

- thorough peer review by experienced researchers in your field

- rapid publication on acceptance

- support for research data, including large and complex data types

- gold Open Access which fosters wider collaboration and increased citations

- maximum visibility for your research: over $100 \mathrm{M}$ website views per year

At BMC, research is always in progress.

Learn more biomedcentral.com/submissions 